\title{
Evaluación electroquímica de una aleación base Ni como electrocatalizador para producir $\mathbf{H}_{2}$
}

\section{Electrochemical evaluation of a $\mathrm{Ni}$ alloy as an electrocatalyst to produce $\mathrm{H}_{2}$}

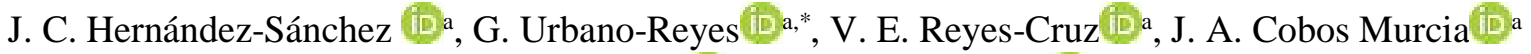 \\ J. C. Juárez-Tapia ${ }^{\circ}$, A. Trujillo-Estrada
}

${ }^{a}$ Área Académica de Ciencias de la Tierra y Materiales, Universidad Autónoma del Estado de Hidalgo, 42184, Pachuca, Hidalgo, México.

${ }^{b}$ Cátedras Consejo Nacional de Ciencia y Tecnología, CONACyT, Ciudad de México, 03940, México.

\begin{abstract}
Resumen
Se presenta un estudio electroquímico para evaluar una aleación de Ni empleada como cátodo y determinar su viabilidad para producir de hidrógeno en un medio electrolítico básico y ácido. Se aplicó un tratamiento a la superficie de la aleación de Ni utilizando

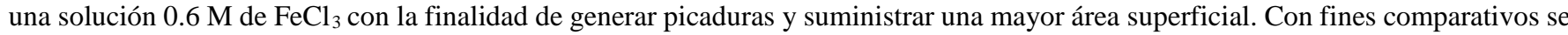
usó también un electrodo de acero inoxidable A304 como cátodo. Se realizaron pruebas de voltamperometría cíclica y cronoamperometría en un rango de potenciales entre -2.0 a $0.0 \mathrm{~V}$, para evaluar la densidad de corriente durante la evolución de hidrógeno. Se determinó que la densidad de corriente obtenida en el medio básico fue menor que en el medio ácido, con ambos electrodos analizados. Mientras que con la aleación de Ni se presentó menores sobrepotenciales para el inicio de la reacción de evolución de hidrógeno, así como una mayor densidad de corriente que con el acero inoxidable A304. Además, se observó un aumento en la densidad de corriente cuando se aplicó el tratamiento a la aleación de $\mathrm{Ni}$, atribuido a un aumento en el área superficial catalítica de la aleación por la generación de picaduras.
\end{abstract}

Palabras Clave:

Aleación de Ni, Electrólisis del agua, Producción de hidrógeno $\left(\mathrm{H}_{2}\right)$, Electrodos DSA, Electrocatalizadores.

\begin{abstract}
An electrochemical study is presented to evaluate a Ni alloy used as a cathode and determine its viability to produce hydrogen in a basic and acid electrolytic medium. The Ni-alloy surface was treated with a $0.6 \mathrm{M} \mathrm{FeCl}_{3}$ solution to generate pitting and provide an increased surface area. For comparison purposes, an A304 stainless steel electrode was also used as a cathode. Cyclic voltammetry and chronoamperometry tests were carried out in a potential range between -2.0 to $0.0 \mathrm{~V}$ to evaluate the current density during hydrogen evolution. The current density obtained in the basic medium was lower than in the acid medium in both electrodes analyzed. For the initiation of the hydrogen evolution, the Ni alloy presented a lower overpotentials reaction and a higher current density than with A304 stainless steel. Furthermore, the increase in current density observed in the treated Ni-alloy is attributed to a rise in the catalytic surface area.
\end{abstract}

Keywords:

Ni alloy, Water electrolysis, Hydrogen production $\left(\mathrm{H}_{2}\right)$, DSA electrodes, Electrocatalysts.

\section{Introducción}

El hidrógeno como portador de energía o vector energético representan una alternativa viable a medio o corto plazo para dar solución a los problemas de crisis energética y el calentamiento global, inducido por la emisión de $\mathrm{CO}_{2}$ (Liu et al., 2018). Algunas valoraciones del potencial de una economía del hidrógeno han centrado su atención en su producción, al ser un aspecto crucial en el desarrollo de un sistema viable. Así, el término economía del hidrógeno responderá a una visión de futuro donde este gas, sea generado de forma limpia y económica. Numerosos grupos de investigación han desarrollado trabajos relevantes en torno a la tecnología del hidrógeno y celdas de combustible, por considerarlas estrategias emergentes y tecnologías prometedoras en la generación de energía limpia y sustentable, con alta densidad de energía y alta eficiencia (Solorsa, 2015). La característica más importante del hidrógeno como vector energético que justifica su estudio es que almacena mayor cantidad de energía por unidad de peso, que cualquier otra sustancia; además de que su combustión produce solamente agua, sin contaminar el ambiente (Isgro, 2015). Además, el hidrógeno es muy importante en diversos procesos y por ello su demanda a nivel mundial es alta. Existen diversos métodos y procesos para la obtención de hidrógeno, sin embargo, la producción de hidrógeno a partir de energías renovables es el objetivo de un sistema energético basado en un ciclo de obtención totalmente limpio (Bañuls, 2017). La

\footnotetext{
*Autor para la correspondencia: gustavo_urbano@uaeh.edu.mx

Correo electrónico: julio_cesar_hdez@outlook.com (Julio Cesar Hernández-Sánchez), gustavo_urbano@uaeh.edu.mx (Gustavo Urbano-Reyes), reyescruz16@yahoo.com (Víctor Esteban Reyes-Cruz), jose_cobos@uaeh.edu.mx (José Ángel Cobos-Murcia), jcjuarez@uaeh.edu.mx (Julio Cesar Juárez-Tapia), ariadnat@gmail.com (Ariadna Trujillo-Estrada).
} 
producción del hidrógeno mediante la electrólisis de agua se considera la forma más limpia para la economía de hidrógeno en un futuro próximo (Ball et al., 2016, citado en Gomez et al., 2018). La electrólisis para la producción de hidrógeno se basa en la separación de los elementos que constituyen el agua, el hidrógeno y el oxígeno, mediante la aplicación de corriente eléctrica. Los iones hidroxilo o iones hidronio son necesarios para que el agua sea conductora. En un medio alcalino las reacciones clave asociadas con la electrólisis del agua son (McCarty et al., 2019):

$$
\begin{array}{ll}
\text { Ánodo } & 2 \mathrm{OH}^{-} \rightarrow 1 / 2 \mathrm{O}_{2}+\mathrm{H}_{2} \mathrm{O}+2 \mathrm{e}^{-} \\
\text {Cátodo } & 2 \mathrm{H}_{2} \mathrm{O}+2 \mathrm{e}^{-} \rightarrow \mathrm{H}_{2}+2 \mathrm{OH}^{-}
\end{array}
$$

Mientras que en un medio ácido las reacciones son:

$$
\text { Ánodo } 2 \mathrm{H}_{2} \mathrm{O} \rightarrow \mathrm{O}_{2}+4 \mathrm{H}^{+}+4 \mathrm{e}^{-}
$$$$
\text { Cátodo } 2 \mathrm{H}^{+}+2 \mathrm{e}^{-} \rightarrow 2 \mathrm{H}_{2}
$$

Por lo tanto, la reacción global en la celda es:

$$
\mathrm{H}_{2} \mathrm{O} \rightarrow \mathrm{H}_{2} \text { (g) }+1 / 2 \mathrm{O}_{2} \text { (g) }
$$

Sin embargo, para que el proceso electrolítico sea más viable es necesario reducir los costos del proceso y del equipamiento o materiales utilizados. Para ello se requiere minimizar el consumo de energía eléctrica utilizada para el proceso de electrólisis, reduciendo el voltaje total empleado en la celda electrolítica (electrolizador). Es decir, se requiere optimizar la eficiencia de la celda electrolítica que permita una resistencia interna menor, con el desarrollo de electrodos de elevada actividad catalítica (electrocatalizadores). Por lo tanto, estos electrodos deben presentar menores sobrepotenciales a las reacciones de desprendimiento de hidrógeno (HER) y oxígeno (OER). Los electrodos deben ser resistentes a la corrosión, tener buena conductividad eléctrica, exhibir buenas propiedades catalíticas y estabilidad estructural (para películas depositadas sobre algún sustrato); así como una mayor área superficial (Isgro, 2015), (Solorsa, 2015). Los catalizadores basados en óxido del metal de transición son rentables y muy activos para OER; sin embargo, sus aplicaciones para catalizar la HER siguen siendo un reto debido a la actividad insatisfactoria y la conductividad electrónica intrínsecamente deficiente (Zhang et al., 2018). Se han empleado diferentes materiales para catalizar la HER (platino, paladio, iridio, rodio) que se depositan sobre un sustrato conductor formando una capa más activa y estable, debido a que estos materiales son muy costosos y escasos en la naturaleza (Solorsa, 2015). También se han utilizado algunos compuestos con metales no preciosos, como aleaciones, calcogenuros, carburos, nitruros, boruros y fosfuros, o materiales libres de metales (Zeng and $\mathrm{Li}$, 2015). Los materiales nanocatalizadores $(2-5 \mathrm{~nm})$ son actualmente soportados en diferentes tipos de carbones, óxidos metálicos y grafenos impurificados o funcionalizados (Desmond et al., 2014, citados por Solorsa, 2015). En un estudio reciente (GonzálezLópez et al., 2018) emplearon electrodos modificados del tipo DSA (ánodos dimensionalmente estables por sus siglas en inglés) fabricados con una lámina de acero inoxidable A304 o Ti como sustrato, aplicando un recubrimiento de $\mathrm{RuO}_{2}$ mediante el método Pechini modificado, con una alta estabilidad química y electroquímica. Además, se probaron diferentes tipos de materiales como cátodos, incluidas placas de titanio (Ti), plata (Ag), aceros inoxidables A304 y A316, barras de grafito (GR), carbono vítreo (VC) y platino (Pt). Los resultados mostraron que la mejor disposición y material de electrodo para la producción catalítica de $\mathrm{H}_{2}$ (g) fue un electrodo DSA de placa de acero inoxidable A304 | RuO2 y el acero inoxidable A304 como cátodo, que era incluso mejor que el platino. García (2012), indicó que los catalizadores basados en Níquel se han mostrado activos tanto para catalizadores anódicos como catódicos, además de mostrar una alta resistencia a la corrosión y una alta estabilidad en el tiempo. La actividad catalítica de los materiales basados en Níquel aumenta cuando se dopan con un segundo metal de transición como Ti, Cr, Mo u otros metales (post-metales) como Sn, Pb, Tl y Cd. La literatura indica que las aleaciones Ni-Mo han mostrado los mejores resultados para el desprendimiento de hidrógeno (HER); por su parte elementos como el Cr proveen a la aleación alta resistencia a la corrosión (García, 2012).

Por lo tanto, esta investigación se basa en el estudio electroquímico de una aleación base $\mathrm{Ni}$ (Fe-Cr-Mo) para emplearla como cátodo en la producción de hidrógeno, en un medio electrolítico básico y un medio ácido. Un electrodo del tipo DSA (acero inoxidable $\mathrm{A} 304 \mid \mathrm{RuO}_{2}$ ) es usado como ánodo para evaluar la densidad de corriente obtenida durante el proceso de la electrólisis del agua para producir hidrógeno.

\section{Procedimiento Experimental}

Todos los experimentos se llevaron a cabo en una celda electroquímica convencional para tres electrodos con una capacidad de $100 \mathrm{ml}$ a temperatura ambiente. El electrodo usado como ánodo en el estudio electroquímico fue un electrodo modificado del tipo DSA, elaborado con una placa de acero inoxidable A304 como sustrato, aplicando un recubrimiento de $\mathrm{RuO}_{2}$ mediante el método Pechini modificado (González-López et al., 2018). Por otro lado, el electrodo de trabajo usado como cátodo fue elaborado de una aleación base níquel, que fue preparada mediante un proceso de fundición y vaciada dentro de un molde de arena en verde en forma de placas (10 X 10 X 1.2 $\mathrm{cm}$ ). Para obtener la composición química deseada (ver Tabla 1) la aleación se preparó en un horno de inducción utilizando níquel electrolítico $(99.9 \% \mathrm{Ni})$ y ferroaleaciones: Fe-Cr (32\% Fe-67\% Cr) y Fe-Mo (38.5\% Fe-61\% Mo). Además, con la finalidad de proporcionar una mayor conductividad eléctrica a la aleación se agregaron algunos elementos como el $\mathrm{Cu}(99.9 \% \mathrm{Cu})$ y Al $(99.9 \%$ $\mathrm{Al})$, en forma de alambre o barra, respectivamente. Finalmente las placas de la aleación se cortan en secciones más pequeñas (2 X 1 X $0.32 \mathrm{~cm}$ ) para utilizarlas como cátodos.

Tabla 1. Análisis químico final de la aleación preparada de Ni (Fe-Cr-Mo) para usarse como cátodo.

\begin{tabular}{cc}
\hline Elemento & $\%$ \\
\hline $\mathrm{Ni}$ & 42.64 \\
$\mathrm{Fe}$ & 20.09 \\
$\mathrm{Cr}$ & 16.75 \\
$\mathrm{Mo}$ & 13.20 \\
$\mathrm{Al}$ & 3.60 \\
$\mathrm{Cu}$ & 2.19 \\
$\mathrm{Si}$ & 0.51 \\
$\mathrm{Mn}$ & 0.22 \\
Balance & 0.80 \\
\hline
\end{tabular}

Por otra parte, con el propósito de suministrar una mayor área superficial al electrodo de trabajo (cátodo) elaborado con la aleación de Ni (Fe-Cr-Mo) mediante la generación de pequeñas picaduras, se le aplicó un tratamiento utilizando una solución 0.6 $\mathrm{M}$ de $\mathrm{FeCl}_{3}$ a temperatura de ebullición durante 30 minutos. Además, un electrodo saturado de Calomel (ESC, E $=0.245$ V vs un electrodo estándar de hidrógeno) fue utilizado como electrodo de referencia en todos los experimentos. Un electrodo de acero inoxidable A304 fue utilizado también como cátodo con fines comparativos, para evaluar la densidad de corriente de la aleación en estudio. La superficie de los electrodos de trabajo (sin tratamiento) fue renovada después de cada experimento puliendo con papel de lija (1200 \#). La solución del electrolito usada como medio básico fue preparada con $10 \mathrm{~g} / \mathrm{L}$ de $\mathrm{NaOH}$ y un $\mathrm{pH}$ de 13.5, 
mientras que la solución del electrolito usada como medio ácido fue preparada de una solución $10 \%$ de $\mathrm{H}_{2} \mathrm{SO}_{4}$ y un pH de 1 . Los estudios electroquímicos se realizaron utilizando un potenciostato/galvanostato Princeton Applied Research 263A ${ }^{\circledR}$ controlado por el software Powersuite 3.0®. Todos los valores de los potenciales se reportaron con respecto al electrodo de calomel (SCE). Se llevaron a cabo experimentos de voltamperometría cíclica en un rango de potenciales entre -2.0 a $0.0 \mathrm{~V}$, iniciados hacia la dirección catódica a una tasa de barrido de $20 \mathrm{mV} \cdot \mathrm{s}^{-1}$. En las pruebas de cronoamperometría se impusieron potenciales fijos en un rango de $-0.5 \mathrm{~V}$ hasta $-2.0 \mathrm{~V}$, durante $360 \mathrm{~s}$ para registrar la densidad de corriente.

\section{Resultados y Discusión}

La Figura 1 muestra las respuestas voltamperométricas obtenidas para el electrodo de trabajo de la aleación Ni (Fe-CrMo) y el acero inoxidable A304, en una solución 10 g/L de $\mathrm{NaOH}$ (Fig. 1a) y una solución al $10 \%$ de $\mathrm{H}_{2} \mathrm{SO}_{4}$ (Fig. 1b). El barrido de potencial se inició en la dirección catódica a una velocidad de 20 $\mathrm{mV} / \mathrm{s}$ a partir del potencial de circuito abierto (OCP). En los voltamperogramas de la Figura 1a se observa un proceso de reducción $\mathrm{C} 1$, que inicia aproximadamente en $-1.4 \mathrm{~V}$, para ambos electrodos. Sin embargo, también se observó que la densidad de corriente catódica obtenida con el electrodo de trabajo de la aleación Ni (Fe-Cr-Mo) fue siempre mayor desde el inicio del proceso de reducción $\mathrm{C} 1$, con respecto a la densidad de corriente obtenida con el electrodo de trabajo de acero inoxidable A304. El incremento de la corriente catódica del proceso de reducción C1, se relacionó con el inicio de la reacción de evolución de hidrógeno, debido a la naturaleza de la solución y los electrodos (Bard and Faulkner et al., 1944), (González-López et al., 2018).
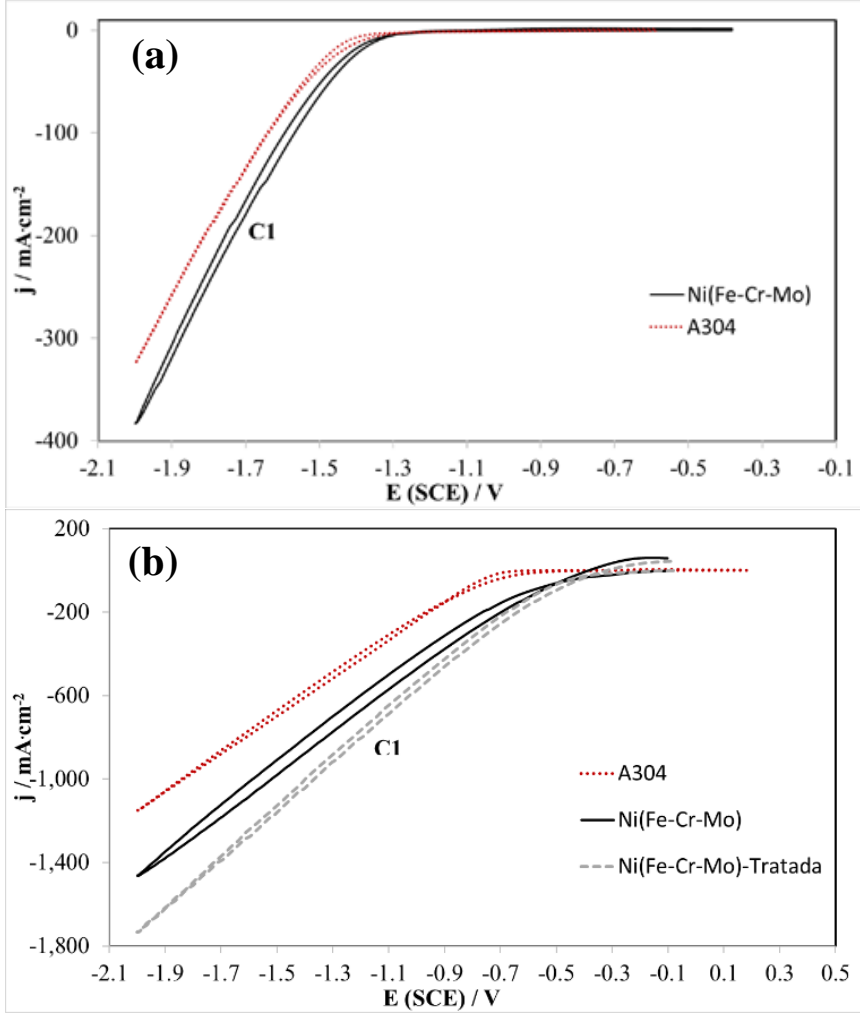

Figura 1: Voltamperogramas obtenidos en dirección catódica de una aleación Ni (Fe-Cr-Mo) con y sin tratamiento y un acero inoxidable A304 en una solución: a) $10 \mathrm{~g} / \mathrm{L}$ de $\mathrm{NaOH}$ y b) $10 \%$ de $\mathrm{H}_{2} \mathrm{SO}_{4}$.
Por otro lado, cuando se empleó la solución $10 \%$ de $\mathrm{H}_{2} \mathrm{SO}_{4}$ (Fig. 1b) los voltamperogramas muestran una mayor densidad de corriente catódica, tanto para la aleación Ni (Fe-Cr-Mo) como para el acero inoxidable A304 usados como cátodos, con relación a cuando se usó la solución $10 \mathrm{~g} / \mathrm{L}$ de $\mathrm{NaOH}$. Este comportamiento fue atribuido a una mayor producción de moléculas de hidrogeno $\left(\mathrm{H}_{2(\mathrm{~g})}\right)$ en un medio ácido (reacción 4), con relación a un medio alcalino (reacción 2). Sin embargo, la densidad de corriente catódica obtenida con el electrodo de trabajo de la aleación $\mathrm{Ni}$ (Fe-Cr-Mo) también fue siempre mayor desde el inicio del proceso de reducción $\mathrm{C} 1$, con relación al acero inoxidable A304. Además, el inicio del proceso asignado a la evolución de hidrógeno (C1), ocurre un poco antes (-0.5 V), con relación al acero inoxidable A304 (-0.7 V). Este comportamiento fue atribuido a una mayor actividad catalítica de la superficie del electrodo de la aleación $\mathrm{Ni}$ (Fe-Cr-Mo) usada como cátodo, debido a los elementos presentes que actúan como electrocatalizadores ( $\mathrm{Ni}$, Mo y $\mathrm{Cr}$ ). Los cuales se encuentran presentes en mayor cantidad en esta aleación y promueve menores sobrepotenciales para las reacciones de desprendimiento de hidrógeno (HER).

Por otra parte, cuando se aplicó el tratamiento a la superficie del electrodo de la aleación Ni (Fe-Cr-Mo) con una solución 0.6 $\mathrm{M}$ de $\mathrm{FeCl}_{3}$, se observa un incremento en la densidad de corriente catódica en el proceso de reducción $\mathrm{C} 1$, con respecto al electrodo de $\mathrm{Ni}$ (Fe-Cr-Mo) sin tratar y acero inoxidable A304. Este incremento en la densidad de corriente fue atribuido a un incremento del área superficial generada por la formación de pequeñas picaduras sobre la superficie de la aleación, lo que promueve una mayor área catalítica. Un análisis más detallado mediante un estudio de cronoamperometría se presenta a continuación, con el propósito de validar el comportamiento de la densidad de corriente bajo los diferentes potenciales impuestos y las diferentes condiciones de los sistemas en estudio.

La Figura 2 muestra los transitorios potenciostáticos obtenidos en un intervalo de potenciales seleccionados entre -0.5 y $-1.5 \mathrm{~V}$ durante $360 \mathrm{~s}$, en un medio básico con la solución $10 \mathrm{~g} / \mathrm{L}$ de $\mathrm{NaOH}$, cuando se usaron los electrodos de trabajo (cátodos) con la aleación de Ni (Fe-Cr-Mo) o el acero inoxidable A304 (Fig. 2a y $2 b$, respectivamente). Se observa que la densidad de corriente relacionada con el inicio de la evolución de hidrógeno comenzó a incrementar a partir de un potencial de aproximadamente $-0.8 \mathrm{~V}$ para ambos electrodos, con un comportamiento casi constante de la densidad de corriente durante todo el tiempo que el potencial fue impuesto. Sin embargo, la densidad de corriente obtenida con el electrodo de trabajo de la aleación Ni (Fe-Cr-Mo) fue siempre mayor con relación al acero inoxidable A304 (ver Tabla 2), tal como se observó en el análisis voltamperométrico realizado.

Tabla 2: Valores de potencial y densidad de corriente (j) obtenida en los electrodos de trabajo estudiados a los $360 \mathrm{~s}$ en un medio electrolítico de $10 \mathrm{~g} / \mathrm{L}$ de $\mathrm{NaOH}$.

\begin{tabular}{|c|c|c|}
\hline $\begin{array}{l}\text { Potencial } \\
(\mathrm{V})\end{array}$ & $\begin{array}{c}\text { Ni (Fe-Cr-Mo) } \\
\text { j }\left(\mathrm{mA} / \mathrm{cm}^{2}\right)\end{array}$ & $\begin{array}{c}\mathrm{A} 304 \\
\mathrm{j}\left(\mathrm{mA} / \mathrm{cm}^{2}\right)\end{array}$ \\
\hline-0.8 & -3.65 & -1.44 \\
\hline-0.9 & -20.7 & -9.61 \\
\hline-1.0 & -65.1 & -36.0 \\
\hline-1.1 & -128.5 & -87.8 \\
\hline-1.2 & -195 & -154 \\
\hline-1.3 & -268 & -231 \\
\hline-1.4 & -345 & -315 \\
\hline-1.5 & -428 & -402 \\
\hline
\end{tabular}

La Figura 3 muestra los transitorios potenciostáticos obtenidos con la solución $10 \%$ de $\mathrm{H}_{2} \mathrm{SO}_{4}$ en un intervalo de potenciales 
seleccionados entre -0.5 y $-2.0 \mathrm{~V}$ durante $360 \mathrm{~s}$, para los electrodos de trabajo de la aleación $\mathrm{Ni}$ (Fe-Cr-Mo) con y sin tratamiento (Fig. 3a y 3c, respectivamente); así como para el acero inoxidable A304 (Fig. 3b). Se observa que la densidad de corriente relacionada con el inicio de la evolución de hidrógeno comienza en un potencial aproximado de $-0.5 \mathrm{~V}$ con los sistemas estudiados, con un comportamiento casi constante durante los potenciales impuestos. Sin embargo, la densidad de corriente obtenida con el electrodo de trabajo de la aleación $\mathrm{Ni}$ (Fe-Cr-Mo) sigue siendo mayor con relación a la respuesta del acero inoxidable A304 (ver Tabla 3), aún sin el tratamiento superficial aplicado. Además, se observa que cuando se usó el electrodo de trabajo de la aleación Ni (FeCr-Mo) la densidad de corriente presentó un comportamiento más uniforme en potenciales más catódicos $(\leq-1.5 \mathrm{~V})$, que con el acero inoxidable A304 (ver Fig. 3a y 3b, respectivamente). Este comportamiento también se atribuyó al comienzo de una degradación de la superficie del electrodo DSA (usado como ánodo), cuando se emplea un electrodo de trabajo de acero inoxidable A304 y se imponen potenciales $\leq-1.5 \mathrm{~V}$. Es decir, esto demuestra que la aleación $\mathrm{Ni}$ (Fe-Cr-Mo) usada como cátodo en conjunto con el electrodo modificado (DSA) usado como ánodo, permite una mejor estabilidad catalítica de la electrólisis del agua para producir hidrógeno de manera estable.

Por otra parte, la Figura 3c muestra que cuando se emplea el electrodo de trabajo de la aleación $\mathrm{Ni}$ (Fe-Cr-Mo) con el tratamiento a la superficie, una mayor densidad de corriente se presentó en todos los potenciales que se impusieron, con respecto a la aleación Ni (Fe-Cr-Mo) sin tratar y el acero inoxidable A304 (ver Tabla 3). Este comportamiento se atribuyó también al incremento en el área superficial catalítica de la aleación Ni (FeCr-Mo) para la electrólisis del agua y la producción de hidrógeno, debido a la generación de pequeñas picaduras con el tratamiento aplicado.
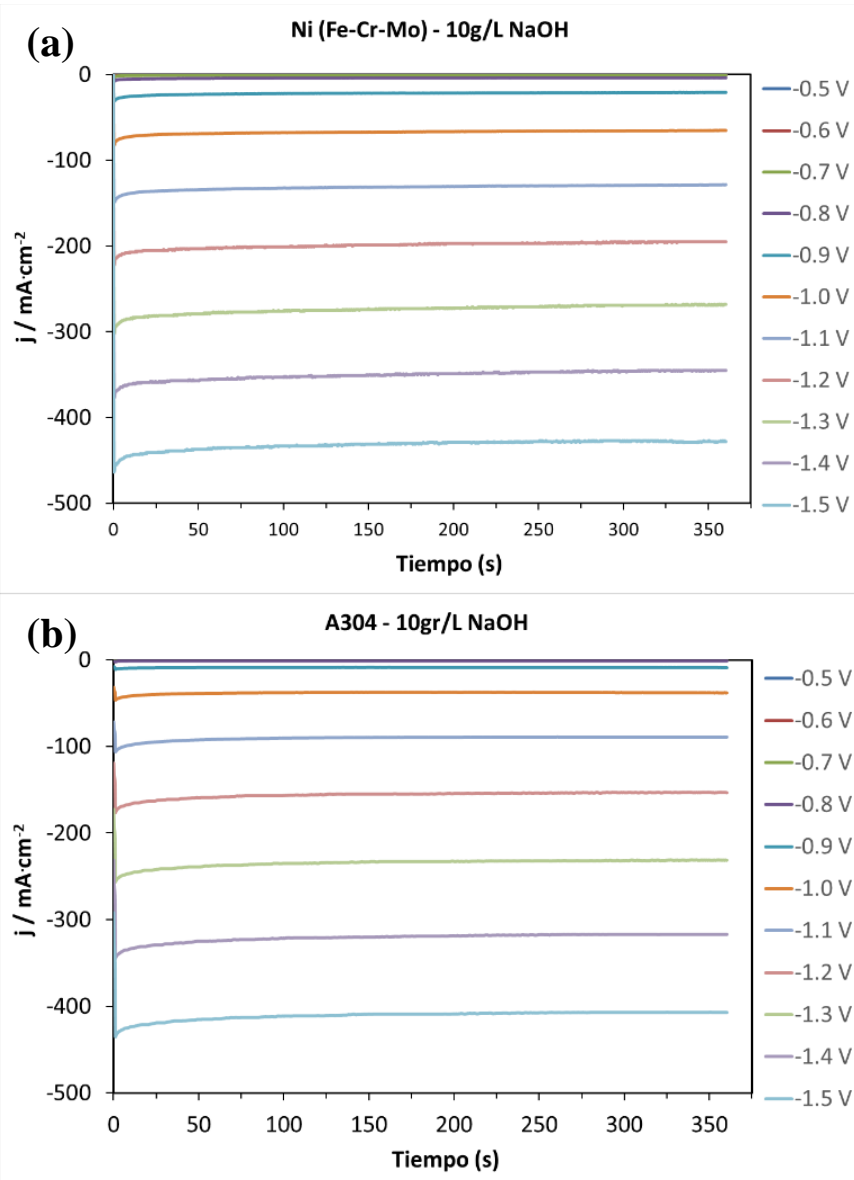

Figura 2: Transitorios potenciostáticos obtenidos durante $360 \mathrm{~s}$ en una solución 10 g/L de $\mathrm{NaOH}$ con la aleación: a) Ni (Fe-CrMo) y b) acero inoxidable A304.
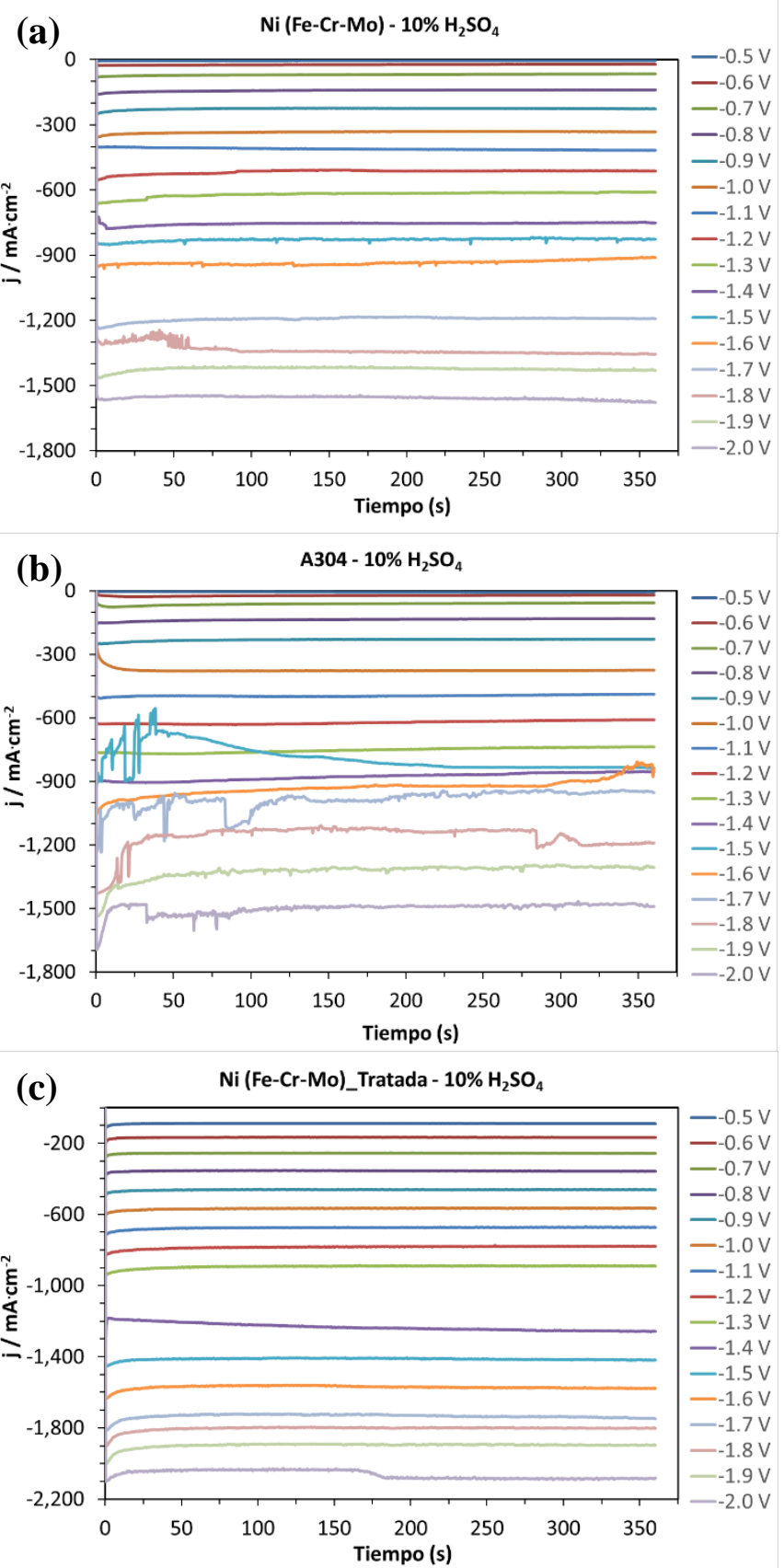

Figura 3: Transitorios potenciostáticos obtenidos durante $360 \mathrm{~s}$ en una solución $10 \%$ de $\mathrm{H}_{2} \mathrm{SO}_{4}$ con la aleación: a) $\mathrm{Ni}$ (Fe-Cr-

$\mathrm{Mo})$, b) acero inoxidable A304 y c) Ni (Fe-Cr-Mo)-Tratada

Sin embargo, también se observó que en el intervalo de potenciales entre $-0.5 \mathrm{~V}$ y $-1.3 \mathrm{~V}$ (Fig. 3c), el incremento de la densidad de corriente fue menor con respecto a los potenciales más catódicos $(\leq-1.4 \mathrm{~V})$, donde se observa un incremento de la densidad de corriente asociada a la producción de hidrógeno de forma masiva. García (2012) indicó que para que ocurra el proceso de electrolisis del agua, es necesario imponer un sobrepotencial (E) superior al potencial termodinámico. Es decir, existe una barrera energética que es necesario superar para que el proceso de electrólisis ocurra, donde la condición de irreversibilidad de la electrolisis obliga a utilizar una energía adicional.

Por último, se pudo observar físicamente que los electrodos usados como cátodos (aleación de $\mathrm{Ni}$ (Fe-Cr-Mo) y acero inoxidable A304) no presentaron cambios o alteraciones en su 
superficie, lo que indica que son resistentes a la corrosión aún bajo las condiciones extremas de pH. Sin embargo, la aleación Ni (FeCr-Mo) usada como cátodo en conjunto con el electrodo modificado (DSA) usado como ánodo, exhiben buenas propiedades catalíticas e integridad estructural durante el proceso de electrólisis del agua, manteniendo una densidad de corriente y de producción de hidrógeno más uniforme, en comparación con el acero inoxidable A304.

Tabla 3: Valores de potencial y densidad de corriente (j) obtenida en los electrodos de trabajo estudiados a los $360 \mathrm{~s}$ en un medio electrolítico de $10 \mathrm{~g} / \mathrm{L}$ de $\mathrm{NaOH}$.

\begin{tabular}{cccc}
\hline $\begin{array}{c}\text { Potencial } \\
(\mathrm{V})\end{array}$ & $\begin{array}{c}\mathrm{Ni}(\mathrm{Fe}-\mathrm{Cr}-\mathrm{Mo}) \\
\mathrm{j}\left(\mathrm{mA} / \mathrm{cm}^{2}\right)\end{array}$ & $\begin{array}{c}\mathrm{A} 304 \\
\mathrm{j}\left(\mathrm{mA} / \mathrm{cm}^{2}\right)\end{array}$ & $\begin{array}{c}\mathrm{Ni}(\mathrm{Fe}-\mathrm{Cr}- \\
\mathrm{Mo})-\mathrm{Tratada} \\
\mathrm{j}\left(\mathrm{mA} / \mathrm{cm}^{2}\right)\end{array}$ \\
\hline-0.5 & -4.8 & -1.7 & -89.7 \\
-0.6 & -21.8 & -10.1 & -167 \\
-0.7 & -66.4 & -46.9 & -256 \\
-0.8 & -139.7 & -121.9 & -357 \\
-0.9 & -227 & -199 & -461 \\
-1.0 & -333 & -375 & -565 \\
-1.1 & -418 & -388 & -673 \\
-1.2 & -513 & -509 & -779 \\
-1.3 & -610 & -737 & -890 \\
-1.4 & -752 & -877 & -1257 \\
-1.5 & -827 & -830 & -1419 \\
-1.6 & -911 & -878 & -1579 \\
-1.7 & -1193 & -953 & -1749 \\
-1.8 & -1357 & -1191 & -1807 \\
-1.9 & -1429 & -1305 & -1897 \\
-2.0 & -1577 & -1469 & -2080 \\
\hline
\end{tabular}

\section{Conclusiones}

El estudio electroquímico mostró que los sobrepotenciales (E) requeridos para el inicio de la electrólisis del agua y la producción de hidrógeno son mayores en un medio básico (aproximadamente $-1.4 \mathrm{~V}$ ) que en un medio ácido (aproximadamente $-0.5 \mathrm{~V}$ ), para los sistemas analizados. Además, la densidad de corriente obtenida durante el proceso de evolución hidrógeno fue mucho menor en el medio básico que con el medio ácido, esto debido a la naturaleza del electrolito y las reacciones catódicas que ocurren (reacción 2 y 4, respectivamente). También se determinó que la aleación de Ni (Fe-Cr-Mo) usada como cátodo presenta menores sobrepotenciales para el inicio de la reacción de evolución de hidrógeno (HER), así como una mayor densidad de corriente obtenida, en comparación con un acero inoxidable A304. Este comportamiento fue atribuido a una mayor actividad catalítica de la superficie del electrodo de la aleación Ni (Fe-Cr-Mo) usada como cátodo, debido a los elementos presentes que actúan como electrocatalizadores (Ni, Mo y Cr). Así mismo, cuando se aplicó el tratamiento a la superficie del electrodo de la aleación Ni (FeCr-Mo) con una solución 0.6 M de $\mathrm{FeCl}_{3}$ se observó un mayor incremento en la densidad de corriente asociado a la producción de hidrógeno, atribuido a un aumento del área superficial catalítica de la aleación Ni (Fe-Cr-Mo) debido a la generación de pequeñas picaduras. Finalmente, los resultados de este estudio muestran que la aleación Ni (Fe-Cr-Mo) usada como cátodo en conjunto con el electrodo modificado (DSA) usado como ánodo, exhiben buenas propiedades catalíticas y estabilidad estructural durante el proceso de electrólisis del agua, para la producción de hidrógeno. Sin embargo, en un estudio a futuro se puede realizar pruebas con tiempos más prologados a una mayor escala para la producción masiva de $\mathrm{H}_{2(\mathrm{~g})}$.

\section{Agradecimientos}

Este trabajo ha sido realizado parcialmente gracias al apoyo de la Universidad Autónoma del Estado de Hidalgo y el Sistema Nacional de Investigadores por la subvención otorgada.

\section{Conflicto de intereses}

Los autores declaran que no existen conflictos de intereses.

\section{Referencias}

Able, B. C., (1945). Nombre del artículo. Nombre de la revista 35, 123-126. DOI: 10.3923/ijbc.2010.190.202

Liu, K. H., Zhong, H. X., Li, S. J., Duan, Y. X., Shi, M. M., Zhang, X. B., ... \& Jiang, Q. (2018). Advanced catalysts for sustainable hydrogen generation and storage via hydrogen evolution and carbon dioxide/nitrogen reduction reactions. Progress in Materials Science 92, 64-111. https://doi.org/10.1016/j.pmatsci.2017.09.001

Solorsa, F. O. (2015). Conversión y Almacenamiento de Energía (CAE). En C. F. Almeraya, 30 Años Impulsando la Electroquímica en México (págs. 180-199). Monterrey, Nuevo León, México: Sociedad Mexicana de Electroquímica. Recuperado el 02 de mayo de 2021 de: http://www.smeq.org.mx/frames/Libro\%20de\%20Aniversario\%20de \%20la\%20SMEQ\%20.pdf

Isgró, M. (2015). El hidrógeno como vector energético, su obtención por electrólisis. Recuperado el 06 de mayo de 2021 de: https://scholar.google.es/scholar?hl=es\&as sdt=0\%2C5\&q=El+hidr\% C3\%B3geno+como+vector+energ\%C3\%A9tico\%3A+su+obtenci\%C3\% B3n+por+electr\%C3\%B3lisis.+La+Plata\%3A+EnAlTecs+SITEMA\&btn $\underline{\mathrm{G}}=$

Bañuls Escrig, I. J. (2018). Caracterización de electrodos de níquel dopados con nanopartí́culas de paladio para la obtención de hidrógeno (Doctoral dissertation, Universitat Politècnica de València). Recuperado el 15 de mayo de 2021 de: https://scholar.google.es/scholar?hl=es\&as_sdt=0\%2C5\&q=Ba\%C3\%B1 uls\%2C+S.+I.+\%28Diciembre+de+2017\%29.+Caracterizaci\%C3\%B3n+ de+electrodos+de+n\%C3\%ADque+dopados+con+nanopart\%C3\%ADcul as+de+paladio+para+la+obtenci\%C3\%B3n+de+hidr\%C3\%B3geno+\%28 Tesis+Doctoral\%29.+Valencia\%2C+Valencia\%2C+Espa\%C3\%B1a\%3A +Universidad+Polit\%C3\%A9cnica+de+Valencia.\&btnG=

Gomez, M. J., Franceschini, E. A., Corti, H. R., \& Lacconi, G. I. (2018). Síntesis y propiedades de electrodos de níquel/grafeno para generación de hidrógeno. Matéria (Rio de Janeiro), 23(2). https://doi.org/10.1590/S1517707620180002.0462

McCarty, R. D., Cox, K. E., \& Williamson, K. D. (2019). Hydrogen: Its Technology and Implications: Hydrogen Properties. CRC Press. https://www.taylorfrancis.com/books/mono/10.1201/9780429487170/hyd rogen-technology-implications-robert-mccarty-kenneth-cox-williamson

Zhang, T., Wu, M. Y., Yan, D. Y., Mao, J., Liu, H., Hu, W. B., ... \& Qiao, S. Z. (2018). Engineering oxygen vacancy on NiO nanorod arrays for alkaline hydrogen evolution. Nano Energy, 43, 103-109. DOI: https://doi.org/10.1016/j.nanoen.2017.11.015

Zeng, M., \& Li, Y. (2015). Recent advances in heterogeneous electrocatalysts for the hydrogen evolution reaction. Journal of Materials Chemistry A, 3(29), 14942-14962. DOI: 10.1039/c5ta02974k

González-López, M. A., Reye-Cruz, V. E., Cobos-Murcia, J. A., VelozRodríguez, M. A., Urbano-Reyes, G., \& Pérez-Labra, M. (2018). Effect of DSA electrode (A304| RuO2) on the electrochemical production of $\mathrm{H} 2$ (g). Int. J. Electrochem. Sci, 13, 10873-10883. DOI:10.20964/2018.11.58

García, J. C. (2012). Producción de hidrógeno mediante electrolizadores. Estado del arte de la tecnología. línea]. Disponible en: http://www. ingenieriaquimica. net/articulos/302-produccion-de-hidrogenomedianteelectrolizadores-estado-del-arte-de-la-tecnologia-electrolizadore. [Accedido: 02-jun-2021].

A. J. Bard and L. R. Faulkner, Electrochemical Methods Fundamentals and Applications, John Wiley \& Sons, Inc., (1944) 605 Third Avenue, New York, NY, United States of America. 